\title{
ON THE MECHANISM OF MUSCLE CONTRACTION (III)
}

\author{
A CONSIDERATION ON "STEADY STATE" \\ IN MUSCULAR CONTRACTION
}

\author{
TORAO NAGAI, YOSHIO TERAYAMA AND MADOKA MAKINOSE \\ Department of Physiology, Sapporo University of Medicine
}

In the preceding papers $(1,2)$, one (T.N.) of the present authors and his co-workers presented their picture of the mechanism of muscular contraction and relaxation. Namely, they concluded that the AM (actomyosin) formation is exothermic and that Varga's data (3), which give an important basis for SzentGyörgyi's theory (4), must be understood as a balance between the contracting reaction resulted from the splitting energy of ATP and the relaxing reaction caused by diminution of the intermolecular cohesion of $\mathrm{AM}$, affected by unspecific ionic milieu. state."

The idea introduced from this view should be nothing but a kind of "steady

In fact, Mugikura (5) proved that the ATP splitting continues even after the time when the model fiber reached a stationary value of contraction. On the superprecipitation of AM solution, the same result has already been reported by Spicer (6). These facts may support immediately the opinion that the muscular contraction is not attributed to the true equilibrium between two states of contractile units but to the dynamic equilibrium or steady state of AM molecule.

The purpose of the present paper is to make clear the practical substance of this idea, "steady state."

\section{THEORETICAL CONSIDERATION}

To facilitate the understanding of the above mentioned situation, the processes of contraction and relaxation were reduced to their elementary reactions.

According to Michaelis and Menten's mode, the reactions were arranged in a list as follows :

$$
\begin{aligned}
& M+S \stackrel{k_{1}}{\longrightarrow} M S \\
& M S \stackrel{k_{2}}{\longrightarrow} M^{*}+P \\
& M^{*}+S \stackrel{k_{3}}{\longrightarrow} M^{*} S
\end{aligned}
$$

Received for publication October 13, 1955.

永井寅男 卦良雄 牧之瀬 望 


$$
\begin{aligned}
M^{*} S \stackrel{k_{4}}{\longrightarrow} M^{*}+P \\
M^{*} \stackrel{k_{5}}{\longrightarrow} M
\end{aligned}
$$

$$
\begin{aligned}
& M: \text { AM in relaxed state } \\
& M^{*}: \text { AM in contracted state } \\
& S: \text { ATP } \\
& P: \text { splitting products } \\
& k: \text { velocity constant in each elementary reaction }
\end{aligned}
$$

During the continuance of the cycle of reactions described above, it can be considered that the concentrations of the intermediate products must reach to and be kept at a stationary value some time after the commencement of those reactions. Then,

$$
d(M S) / d t=0, \quad d\left(M^{*} S\right) / d t=0, \quad d\left(M^{*}\right) / d t=0 .
$$

According to the "law of mass-action" and eq. (6)

$$
\begin{aligned}
k_{1}(M)(S) & =k_{2}(M S) \\
k_{3}\left(M^{*}\right)(S) & =k_{4}\left(M^{\star} S\right) \\
k_{5}\left(M^{*}\right) & =k_{2}(M S)
\end{aligned}
$$

While, if the concentration of total contractile unit is represented by the $\operatorname{sign}\left(M_{0}\right)$,

$$
\left(M_{0}\right)=(M)+(M S)+\left(M^{*}\right)+\left(M^{*} S\right)
$$

Then, the degree of contraction, $\alpha$, may be assumed as follows.

$$
\alpha=\frac{\left(M^{*}\right)+\left(M^{*} S\right)}{\left(M_{0}\right)}
$$

Combining equations (7), (8), (9), (10) and (11), the relation between the degree of contraction, $\alpha$, and the concentration of ATP, $(S)$, can be obtained as follows.

$$
\alpha=\frac{(S)^{2}+\frac{k_{4}}{k_{3}}(S)}{(S)^{2}+\frac{k_{4}}{k_{3}}\left(1+\frac{k_{5}}{k_{2}}\right)(S)+\frac{k_{4} k_{5}}{k_{1} k_{3}}}
$$

As shown in eq. (12), the degree of contraction is not a problem of simple equilibrium. However, it can be treated as an apparent equilibrium. That is to say, when the amount of the substrate is so great that the change of its concentration by enzyme activity can be ignored, the degree of contraction is higher with increase of substrate concentration as shown in fig. 1.

According to the same mathematical treatment as described above, the rate of ATP splitting can be introduced as follows. 


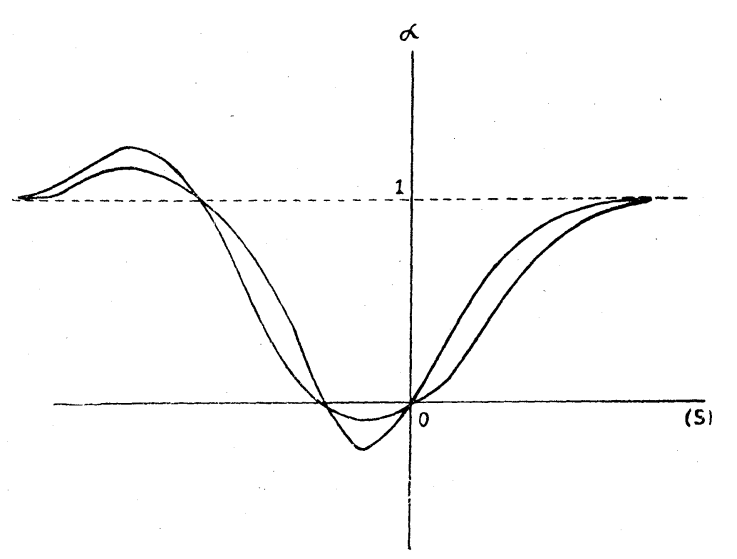

FIG. 1. Theoretical curve representing the relation between the grade of contraction, $\alpha$, and the concentration of ATP, $(S)$.

$$
d(P) / d t=k_{2}(M S)+k_{4}\left(M^{*} S\right)=\left\{\frac{(S)^{2}+\frac{k_{4} k_{5}}{k_{3}}(S)}{(S)^{2}+\frac{k_{4}}{k_{3}}\left(1+\frac{k_{5}}{k_{2}}\right)(S)+\frac{k_{4} k_{5}}{k_{1} k_{3}}}\right\}\left(M_{0}\right)
$$

\section{DISCUSSION}

Fig. 1 shows the theoretical relationship between the degree of contraction of AM-system and the concentration of ATP. In this figure, the curve bears practical meaning only within the range of $(\mathrm{ATP})>0$. In this range, the curve shows two typical shapes, namely a sigmoid form and a hyperbolic one. These two curves equally approach to the line of $\alpha=1$ gradually with increase of ATP concentration.

Lajtha (7) and Weber (8) reported the relationship between ATP contraction of glycerinated muscle fiber and concentration of ATP. Furthermore, the relation between ATPase activity of AM (the rate of ATP splitting) and ATP concentration was reported also by Weber (8). These experimental data bear a close resemblance with the present theoretical results. That is to say, the present equations are quite satisfactory to explain the experimental data except the phenomenon of "Eigenhemmung" the inhibition of contraction or ATP splitting by high concentration of ATP itself.

The discrepancy between the ATPase activity and the grade of superprecipitation within the range of $30^{\circ} \sim 40^{\circ} \mathrm{C}$., as was stated in the preceding paper (2) can also be explained by these equations. Makinose (9) demonstrated that the rate of extension of glycerinated muscle fiber increases suddenly when temperature rises above $30^{\circ} \mathrm{C}$, and pointed out that there are good reasons to suppose that the rate of relaxing process increases suddenly when temperature passes over this point. In eqs. (12) and (13), one can easily find that the essential 
difference between these two equations is the presence of $k_{5}$ in the numerator of eq. (13). The rate of relaxing process is entirely due to this factor, $k_{5}$. This situation will be sufficiently convincing for illustration of the discrepancy between ATPase activity and contraction observed between $30^{\circ} \sim 40^{\circ} \mathrm{C}$.

According to the present idea, the cycle of reactions is performed even while the fiber or AM-superprecipitate is maintaining a given grade of contraction, i.e. the $\mathrm{AM}$ in relaxed state is transforming into contracted state uninterruptedly and the inverse reactions are behaving likewise. Then the ATP splitting is continued during contraction and, in company with this reaction, heat production should be exhibited. This heat production, supposed on model fiber in contracted state, can be considered as an illustration for "heat of maintenance" in living muscle emphasized by Hill (10). Further, this point is expected to be an opening to quantitative analysis in this field.

In the present cycle, eqs. (3) and (4) were introduced in consequence of the data reported by Mugikura (5) that AM system in contracted state shows also ATP splitting at almost the same rate as occurs during the contraction. Then, the present authors considered that AM molecule in contracted state $\left(M^{*}\right)$ possesses appropriate ATPase activity. However, if these equations can be ignored, i.e. if the ATP splitting by contracted AM molecule can be disregarded under certain conditions, then eqs. (12) and (13) can be written in more simplified form. Then, the curves induced by these simplified equations show only hyperbolas.

In constructing the equations to represent the present cycle, the present authors abbreviated the counter reactions in eqs. (1) and (3). Even regarding these counter reactions, one may find no essential difference from the present result but only complicated literal coefficient in eqs. (12) and (13).

As described in the present paper, some experimental facts which could not be solved by the old views can be explained by the present authors' idea of "steady state," although the illustrations are only qualitative. Hill (10) and Weber (8) stated that the muscular contraction is not a simple equilibrium reaction and that it should be considered as a kind of steady state cycle. Polissar (11), on the other hand, introduced the idea of steady state cycle, between contracted state and relaxed state in living muscle apriori, and demonstrated that some data obtained by Hill and others can be explained by means of this idea. The present idea is not certainly so complete as to enable an explanation of all phenomena in muscular contraction. But it is believed that the direction of the ideas and the treatment of the phenomenon exhibited in the present paper, suggest many possibilities on further researches in this field.

Thermodynamic analysis may be considered as the first step for understanding the materials in the molecular state. Accordingly, it is important to produce data on a quantitative analysis of the steady state cycle and, furthermore, it is desirable that high molecular physical studies be brought nearer to and combined with studies on muscular contraction.

\section{SUMMARY}

1) Standing on the experimental facts stated in the two immediately pre- 
ceding papers of this series, a "steady state cycle" was proposed as a mechanism of muscular contraction.

2) The practical substance of the "steady state cycle" in actomyosin system was illustrated.

3) It is exhibited that many phenomena in living or model muscle can be explained advantageously by the present mechanism.

4) The further development of the present idea was discussed.

\section{REFERENCES}

1. NAgaI, T. ET aL. Jap. J. Physiol. 5 : Supp. 355, 1956.

2. NAGAI, T. ET AL. Jap. J. Physiol. 5 : Supp. 363, 1956.

3. VARGA, L. Enzymologia 14:196, 1950;14:212, 1950.

4. Szent-Györgyi, A. Chemistry of Muscular Contraction. New York: Academic Press, 1951.

5. Mugikura, H. Sap. Med. J., in Press.

6. SPICER, S. S. J. Biol. Chem. 199 : 301, 1952.

7. LAJTHA, A. Enzymologia 14: 254, 1950.

8. Weber, H. H. Advances in Protein Chemistry VII : 161, 1952.

9. Makinose, M. ANd Nagai, T. Jap. J. Physiol. 5: 160, 1955.

10. HILl, A. V. Nature $167: 377,1951$.

11. Polissar, M. J. Am. J. Physiol. 168: 776, 1952. 